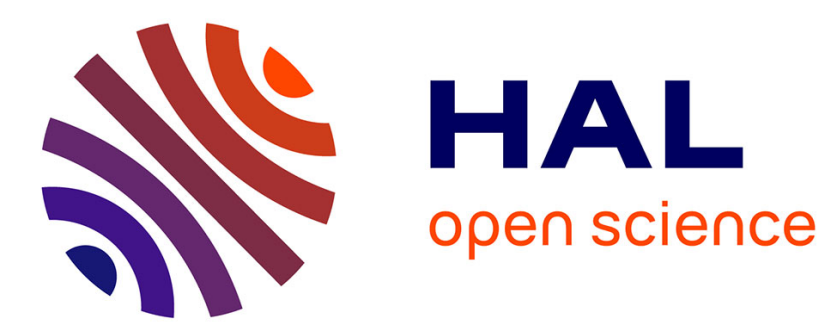

\title{
Analytical Framework for Joint Mode Selection and Power Allocation for Full Duplex D2D Network
}

\author{
Hussein Chour, Oussama Bazzi, F. Bader, Youssef Nasser
}

\section{To cite this version:}

Hussein Chour, Oussama Bazzi, F. Bader, Youssef Nasser. Analytical Framework for Joint Mode Selection and Power Allocation for Full Duplex D2D Network. IEEE Wireless Communications and Networking Conference (WCNC'2019), Apr 2019, marrakech, Morocco. 10.1109/WCNC.2019.8885736 . hal-02002842

\section{HAL Id: hal-02002842 \\ https://hal.science/hal-02002842}

Submitted on 22 Apr 2019

HAL is a multi-disciplinary open access archive for the deposit and dissemination of scientific research documents, whether they are published or not. The documents may come from teaching and research institutions in France or abroad, or from public or private research centers.
L'archive ouverte pluridisciplinaire HAL, est destinée au dépôt et à la diffusion de documents scientifiques de niveau recherche, publiés ou non, émanant des établissements d'enseignement et de recherche français ou étrangers, des laboratoires publics ou privés. 


\title{
Analytical Framework for Joint Mode Selection and Power Allocation for Full Duplex D2D Network
}

\author{
Hussein CHOUR ${ }^{1,2}$, Oussama BAZZI ${ }^{2}$, Faouzi BADER ${ }^{1}$, and Youssef NASSER ${ }^{3}$ \\ ${ }^{1}$ CentraleSupélec/IETR, CentraleSupélec Campus de Rennes, 35510 Cesson-Sévigné, France \\ ${ }^{2}$ Physics and Electronics Department, Faculty of Science 1, Lebanese University, Beirut, Lebanon \\ ${ }^{3}$ ECE Department, American University of Beirut, Bliss Street, Beirut, Lebanon, \\ Email:\{hussein.chour@supelec.fr, faouzi.bader@supelec.fr, yn10@aub.edu.lb, obazzi@ul.edu.lb, \}
}

\begin{abstract}
In this paper, a simple and accurate analytical framework for the duplex mode selection and the power allocation scheme for full duplex (FD) device-to-device (D2D) communications underlaying wireless cellular network is proposed. We first aim at maximizing the D2D links rate by properly selecting the duplex mode of the D2D pairs and allocating the power of the users while fulfilling the quality-of-service $(\mathrm{QoS})$ of the cellular users (CUEs). Since the resulting optimization problem is non-convex, we derive a first-order optimal solution by leveraging an interesting optimization tool named as sequential convex optimization theory. We also discuss how to obtain an efficient sub-optimal solution in term of complexity and accuracy by deriving the optimal power ratio between the D2D devices assuming an interference limited system. The simulations have shown more than $95 \%$ accuracy of the proposed approaches and provided great insights on the solution design parameters taking into account the $\mathrm{CU}$ location and the self-interference threshold.

Index Terms-full-duplex transmission, device-to-device (D2D) communication, optimal power allocation, duplex mode selection
\end{abstract}

\section{INTRODUCTION}

With the rapid growth in the users' data traffic demands and the scarcity of the radio spectrum [1], the need of new technologies which use the spectrum efficiently and meet the users' data requirements has become an essential part in the future cellular network (5G). In this context, device-to-device (D2D) communication and Full Duplex (FD) technologies are proposed to improve the cellular spectrum utilization and the users' experience in a cellular network. D2D allows two nearby devices to initiate a direct link without base station (BS) participation or with limited participation [2]. On the other hand, FD allows a pair of devices to simultaneously transmit and receive in the same frequency band at the same time slot [3].

The exploitation of the FD scheme in D2D technology can further improve the cellular spectrum efficiency and the users throughput due to the short distance property of the D2D communication [4]. However, the practical full duplex transceivers add new challenges for the D2D communication. For instance, the existing FD devices cannot perfectly eliminate the self interference (SI) imposed on the receiver by the node's own transmitter [3]. Thus, the residual self interference (RSI) which is tightly related to the transmitter power value highly affects the performance of FD transmission. In addition, using the FD technique creates additional interference in the network which may degrade its performance when compared with the half duplex (HD) transmission. Accordingly, the power allocation strategy and the selection of the D2D transmission mode are very important problems to be tackled.

The power optimization problem of an isolated full duplex D2D (FD-D2D) pair underlay cellular network was tackled in [5]. Built on this, the authors of [6] derived a convex optimization problem to maximize the rate of FD-D2D link while satisfying the minimum rate requirement of the cellular users. However, neither [5] or [6] provided an analysis on the best D2D transmission mode. Moreover, the solution therein is rather algorithmic. Without addressing the power allocation problem, the authors of [7] derived and analysed a closed form expression for the Ergadic sum rate of a FDD2D network. The mode selection problem of a FD-D2D link was tackled in [8]-[10]. Using stochastic geometry the authors of [8] proved that by properly switching between HD and FD, the system spectrum efficiency of a FD-D2D pair can be improved. In [9], the mode selection problem was investigated for an overlay and underlay FD-D2D enabled cellular network. However, the optimal power allocation was not discussed either in [8] nor in [9]. In [10] the duplex mode selection is investigated considering a D2D underlaying scenario and aiming to maximize the rate of the cellular user with a minimum constraint on the D2D users. Since the main goal of the D2D technology is to alleviate the load from the cellular network, it is more interested to study the performance of FD-D2D communication when the goal is to maximize the D2D rate while fulfilling the QoS of the CUE. In an interference limited scenario, the latter has a non-convexity structure and thus it will lead to a much challenging tasks. In addition, no closed form expression of the optimal power allocation was provided.

In this paper, we analytically investigate both the duplex mode selection and the power allocation problem of a FD-D2D underlaying cellular network. In particular, we provide two approaches to solve the power allocation problem. The first is based on the sequential optimization theory (SCO), [11][13], which is iterative based solution. In the second solution, we develop an analytical framework which yields to a suboptimal solution but with high accuracy and low complexity. 
Moreover, it provides a closed form expression for the optimal power allocation and an upper bound condition for the usage of full duplex.

The rest of this paper is organized as follows. Section II describes the system model and formulates the optimization problem. Section III and IV derive the optimal solution and section V provides the simulation results. Finally, the conclusion is drawn in section VI.

\section{SYSTEM MODEL AND PROBLEM FORMULATION}

We consider a D2D communication underlaying cellular network where $M$ D2D pairs coexist with $M$ cellular users. Each D2D pair $D 2 D_{j}$ consists of two nearby devices denoted as $D_{1}^{j}$ and $D_{2}^{j}$ and they have the capability to operate on both Full duplex and Half duplex modes, while the $\mathrm{CU}$ is assumed to be operated only in HD mode. Moreover, the D2D pair shares the uplink resources of the CU. In particular, UL resources sharing is considered since the UL resources are underutilized comparing to that of downlink. Furthermore, sharing the UL spectrum only affects the BS and incurred interference can be handled by BS coordination. We also assume that $D 2 D_{j}$ can reuse only the uplink channel of $C U_{j}$ and the CUs are allocated orthogonal channels. In other word, the focus in this work is on the mode selection and power allocation and not on the channel assignment step.

An instance of this network is depicted in Fig.1 in which two D2D pairs coexist with two cellular users $(M=2)$. The first D2D pair $D 2 D_{1}$ depicts the case of FD D2D communication while $D 2 D_{2}$ depicts the HD-D2D mode. The channel power gain between the $j$ th cellular user and the BS is denoted as $g_{c, b s}^{j}$ while the channel power gains between $D_{1}^{j}$ and $D_{2}^{j}$ and between $D_{2}^{j}$ and $D_{1}^{j}$ are denoted as $g_{d}^{j}$. The interference channel power gains from $D_{1}^{j}$ and $D_{2}^{j}$ to BS are respectively denoted by $h_{d 1, b s}^{j}$ and $h_{d 2, b s}^{j}$, while $h_{c, d 1}^{j}$ and $h_{c, d 2}^{j}$ denote the interference channel power gains from the $j$ th cellular user to $D_{1}^{j}$ and $D_{2}^{j}$ respectively. All direct/interference channels are assumed to be zero-mean complex Gaussian random variables (i.e., channels are expressing Rayleigh fading) with variance $l_{i j}^{-\alpha}$, where $i \in\{c ; d 1 ; d 2\}, j \in\{b s ; d 1 ; d 2\}, i \neq j, l_{i j}$ denotes the distance between the nodes $i$ and $j$, and $\alpha$ denotes the path loss exponent.

The channels $h_{11}^{j}$ and $h_{22}^{j}$ are the self interference channels at $D_{1}^{j}$ and $D_{2}^{j}$. They can be modeled as complex Gaussian random variables with zero-mean and variance $\eta P_{d 1}^{j}$ and $\eta P_{d 2}^{j}$ respectively [5], [6], [8]-[10], [14], where $P_{d 1}^{j}$ and $P_{d 1}^{j}$ are respectively the transmission power of $D_{1}^{j}$ and $D_{2}^{j}, \eta$ denotes the SI cancellation capability of each D2D device. Hence, the residual SI power at $D_{1}^{j}$ and $D_{2}^{j}$ are respectively $\eta P_{d 1}^{j}$ and $\eta P_{d 2}^{j}$.

\section{A. Full duplex D2D link}

In the FD D2D communication mode, both $D_{1}^{j}$ and $D_{2}^{j}$ reuse the spectrum of $C U_{j}$ to communicate with each other. Denote by $N_{0}$ the power of additive white Gaussian noise and let $P_{c}^{j}$ be the transmission power of the $j$ th cellular user, the received signal to interference plus noise ration (SINR) at BS,

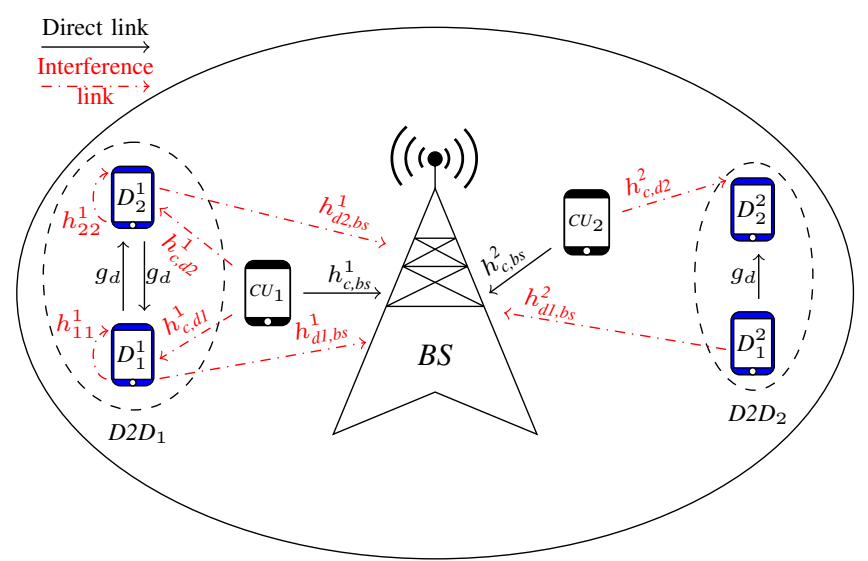

Fig. 1. A full duplex and half duplex D2D pairs sharing the UL resources of the cellular users.

$D_{1}^{j}$ and $D_{2}^{j}$ when $D 2 D_{j}$ shares the spectrum of $C U_{j}$ can be respectively expressed as

$$
\begin{array}{r}
\Gamma_{b s}^{j}=\frac{P_{c}^{j} g_{c, b s}^{j}}{P_{d 1}^{j} h_{d 1, b s}^{j}+P_{d 2}^{j} h_{d 2, b s}^{j}+N_{0}} \\
\Gamma_{\mathrm{FD}, d 1}^{j}=\frac{P_{d 2}^{j} g_{d}^{j}}{P_{c}^{j} h_{c, d 1}^{j}+\eta P_{d 1}^{j}+N_{0}} \\
\Gamma_{\mathrm{FD}, d 2}^{j}=\frac{P_{d 1}^{j} g_{d}^{j}}{P_{c}^{j} h_{c, d 2}^{j}+\eta P_{d 2}^{j}+N_{0}}
\end{array}
$$

Let $B_{j}$ be the bandwidth of the $j$ th CU spectrum. Applying Shannon theorem, the rate of the $j$ th D2D when FD mode has been selected can be expressed as:

$$
R_{\mathrm{FD}}^{j}=B_{j} \log _{2}\left(1+\Gamma_{\mathrm{FD}, d 1}^{j}\right)+B_{j} \log _{2}\left(1+\Gamma_{\mathrm{FD}, d 2}^{j}\right)
$$

\section{B. Half duplex D2D link}

For fair comparison with the FD mode, we assume that $D_{1}^{j}$ and $D_{2}^{j}$ are operating on two equally orthogonal portions of $B_{j}$ during the transmission. Thus, the received SINRs at BS from each couple $\left(D 2 D_{j}, C U_{j}\right)$ remains the same as in the FD mode, i.e., eq(1). However, and since in HD mode the RSI does not longer exist, the received SINR at $D_{1}^{j}$ and $D_{2}^{j}$ when $D 2 D_{j}$ is operating in HD changed to the follows:

$$
\begin{aligned}
\Gamma_{\mathrm{HD}, d 1}^{j} & =\frac{P_{d 2}^{j} g_{d}^{j}}{P_{c}^{j} g_{c, d 1}^{j}+N_{0}} \\
\Gamma_{\mathrm{HD}, d 2}^{j} & =\frac{P_{d 1}^{j} g_{d}^{j}}{P_{c}^{j} g_{c, d 2}^{j}+N_{0}}
\end{aligned}
$$

Again by using Shannon theorem, the HD-D2D link capacity of $D 2 D_{j}$ is given by

$$
R_{H D}^{j}=\frac{1}{2} B_{j} \log _{2}\left(1+\Gamma_{\mathrm{HD}, d 1}^{j}\right)+\frac{1}{2} B_{j} \log _{2}\left(1+\Gamma_{\mathrm{HD}, d 2}^{j}\right)
$$

\section{Problem formulation}

The main goal of D2D technology is to offload a portion of the data traffic from the cellular network. Thus, in this paper, we aim to maximize the total D2D capacity while satisfying the QoS of the cellular users. Since each D2D pair may operate 
either in HD or FD mode, two maximization problem must be studied.

Let $R_{\text {min }}^{j}$ be the minimum data rate for the $j$ th cellular user, and denote by $\mathbf{P}=\left[\mathbf{p}_{j}\right]_{M \times 3}$ the matrix of the users' transmission power with $\mathbf{p}_{j}=\left(P_{d 1}^{j}, P_{d 2}^{j}, P_{c}^{j}\right)$ being the transmission power of the couple $\left(D 2 D_{j}, C U_{j}\right)$, the D2D capacity maximization problem in the HD and the FD modes can be respectively formulated as follows:

$$
\begin{aligned}
& \boldsymbol{P 1}: \max _{\mathbf{P}} R_{d 2 d}^{H D}=\sum_{j=1}^{M} R_{H D}^{j} \text { s.t. } \mathbf{P} \in \Phi \\
& \boldsymbol{P 2}: \max _{\mathbf{P}} R_{d 2 d}^{F D}=\sum_{j=1}^{M} R_{F D}^{j} \text { s.t. } \mathbf{P} \in \Phi \\
& \Phi=\left\{\Gamma_{b s}^{j} \geq \gamma_{\min }^{j}=2^{R_{\min }^{j}}-1, j \in\{1, \ldots, M\}\right. \\
& \left.0 \leq P_{i}^{j} \leq P_{\max }^{i}, i \in\{d 1, d 2, c\}, j \in\{1, \ldots, M\}\right\}
\end{aligned}
$$

where $P_{\max }^{i}$ is the maximum transmission power of a transmitter $i$. Solving $\boldsymbol{P 1}$ is the target of the following section.

\section{POWER ALLOCATION AND DUPLEX MODE SELECTION}

In this section, we focus on finding the best duplex mode and power allocation for all the users in the network with the assumption that the BS has perfect knowledge about the channel state information for both D2D users and CUs.

\section{A. Problem reformulation}

Observing that the couples $\left(D 2 D_{j}, C U_{j}\right), j \in\{1, \ldots, M\}$, are independent from each others, the maximization problems $\boldsymbol{P 1}$ and $\boldsymbol{P 2}$ can be respectively rewritten as follows:

$$
\begin{gathered}
\boldsymbol{P 1}: R_{d 2 d}^{H D}=\sum_{j=1}^{M} \max _{\mathbf{p}_{j}} R_{H D}^{j} \text { s.t. } \mathbf{p}_{j} \in \Phi \\
\boldsymbol{P 2}: R_{d 2 d}^{F D}=\sum_{j=1}^{M} \max _{\mathbf{p}_{j}} R_{F D}^{j} \text { s.t. } \mathbf{p}_{j} \in \Phi
\end{gathered}
$$

Moreover, depending on the channel situation of each couple $\left(D 2 D_{j}, C U_{j}\right)$, the HD or the FD mode may be the optimal duplex mode. Therefore, the maximum D2D capacity can be achieved by optimizing the power of each transmission mode and then selecting the duplex mode with higher rate for each $\left(D 2 D_{j}, C U_{j}\right)$. Thus the total D2D capacity maximization problem can be reformulated as follows:

$$
\begin{aligned}
& \boldsymbol{P 3}: R_{d 2 d}=\sum_{j=1}^{M} \max \left(\mathbf{P A}_{F D}^{j}, \mathbf{P A}_{H D}^{j}\right) \\
& \mathbf{P A}_{F D}^{j}: \max _{\mathbf{p}_{j}} R_{F D}^{j} \text { s.t. } \mathbf{p}_{j} \in \Phi \\
& \mathbf{P A}_{H D}^{j}: \max _{\mathbf{p}_{j}} R_{H D}^{j} \text { s.t. } \mathbf{p}_{j} \in \Phi
\end{aligned}
$$

with $\mathbf{P A}{ }_{F D}^{j} / \mathbf{P A}_{H D}^{j}$ being the power allocation problem for the couple $\left(D 2 D_{j}, C U_{j}\right)$ operating in FD/HD mode. The couples $\left(D 2 D_{j}, C U_{j}\right)$ are independent, and thus in the following, and for ease of notation we derive the solution of $\boldsymbol{P} \mathbf{3}$ for a sample pair $(D 2 D, C U)$ i.e., we omit the $j$ superscript from all the notation. The solution for any pair will be the same.

It can be observed that $\boldsymbol{P 3}$ is a non-concave problem, since both $R_{F D}$ and $R_{H D}$ are not concave function with p. Thus the global optimal solution of $\boldsymbol{P 3}$ is very hard to achieved. Therefore, in the following we present two suboptimal solutions. The first one obtains a first order optimal solution by using the sequential convex optimization theory (SCO). The SCO is an iterative based solution, and thus it may require high number of iteration to converge in some situation. In addition, it does not give a closed form solution. Therefore, to further reduce the complexity and derive a closed form solution we propose a new sub-optimal solution that finds the optimal power ratio between the users in the SIR domain which in turn leads to a high efficient sub-optimal solution of $\boldsymbol{P 3}$ in term of complexity and accuracy. Note that, even if it not mathematically proved, in most of cases the SCO theory finds the global optimal solution of the problem as reported in [15] [16]. Thus, the SCO method will be used to benchmark the results of our proposed method.

\section{B. Sequential convex optimization method}

In this section we solve the optimization problem defined in $\boldsymbol{P 3}$ by using the sequential convex optimization method. The SCO technique is an iterative algorithm which finds local optima of a non-convex maximization problem with objective $f$, by solving a sequence of simpler problems with convex objectives $\left\{f_{i}\right\}_{i}$. Moreover, SCO is guaranteed to converge to a first-order optimal solution of $\boldsymbol{P 3}$ when at each iteration $i$ the following three properties are satisfied [12, Section IV].

- $f_{i}(x) \leq f(x), \forall x$;

- $f_{i}\left(x_{i-1}^{*}\right)=f\left(x_{i-1}^{*}\right)$;

- $\nabla f_{i}\left(x_{i-1}^{*}\right)=\nabla f\left(x_{i-1}^{*}\right)$;

where $x_{i-1}^{*}$ is the maximizer of $f_{i-1}$. Hence,the key point in SCO is to find a simpler objective $\left\{f_{i}\right\}_{i}$ which fulfill the above three properties. In the following we show how to apply SCO in $P 3$.

Since both $R_{d 2 d}^{F D}$ and $R_{d 2 d}^{H D}$ have the same function structure we will demonstrate how to derive the first-order optimal solution only for the FD mode. Now, using the logarithm properties $R_{d 2 d}^{F D}$ is reshaped as follows:

$$
\begin{aligned}
R_{F D}= & B\left[\log _{2}\left(P_{c} g_{c, d 1}+\eta P_{d 1}+N_{0}+P_{d 2} g_{d}\right)\right. \\
& +\log _{2}\left(P_{c} g_{c, d 2}+\eta P_{d 2}+N_{0}+P_{d 1} g_{d}\right) \\
& -\log _{2}\left(P_{c} g_{c, d 1}+\eta P_{d 1}+N_{0}\right) \\
& \left.-\log _{2}\left(P_{c} g_{c, d 2}+\eta P_{d 2}+N_{0}\right)\right] \\
= & r_{f d}^{+}(\mathbf{p})-r_{f d}^{-}(\mathbf{p})
\end{aligned}
$$

observe that $r_{f d}^{+}$and $r_{f d}^{-}$are concave functions and recall that any concave function is upper bounded by its first-order Taylor expansion at any point. Therefore, for any given power vector $\mathbf{p}^{t}$ we can approximate (15) as follows:

$$
R_{F D} \geq r_{f d}^{+}(\mathbf{p})-\left[r_{f d}^{-}\left(\mathbf{p}^{t}\right)+\left(\left.\nabla_{\mathbf{p}} r_{f d}^{-}\right|_{\mathbf{p}=\mathbf{p}^{t}}\right)^{T}\left(\mathbf{p}-\mathbf{p}^{t}\right)\right]
$$


Hence, (16) is lower bound of the utility in (15). Moreover, since the lower bound in (15) is tight when evaluated in $\mathbf{p}^{t}$, (16) is equal to (15) for $\mathbf{p}=\mathbf{p}^{t}$. Next, by using the linearity property of the gradient it is easily verified that $\nabla((15))=$ $\left.\nabla((16))\right|_{\mathbf{p}=\mathbf{p}^{t}}$. Thus, all the above requirements are satisfied and by using this relaxation the convergence of SCO to the first order optimal solution is guaranteed.

Similarly, the approximation of $R_{H D}$ can be derived as:

$$
R_{H D} \geq r_{h d}^{+}(\mathbf{p})-\left[r_{h d}^{-}\left(\mathbf{p}^{t}\right)+\left(\left.\nabla_{\mathbf{p}} r_{h d}^{-}\right|_{\mathbf{p}=\mathbf{p}^{t}}\right)^{T}\left(\mathbf{p}-\mathbf{p}^{t}\right)\right]
$$

wherein $r_{h d}^{+}=\frac{1}{2} B\left(\log _{2}\left(P_{c} g_{c, d 1}+N_{0}+P_{d 2} g_{d 2, d 1}\right)+\right.$ $\log _{2}\left(P_{c} g_{c, d 2}+N_{0}+P_{d 1} g_{d 1, d 2}\right)$ and $r_{h d}^{-}=\frac{1}{2} B\left(\log _{2}\left(P_{c} g_{c, d 1}+\right.\right.$ $\left.\left.N_{0}\right)+\log _{2}\left(P_{c} g_{c, d 2}+N_{0}\right)\right)$.

Note that both (16) and (17) are convex function (since they are summation of concave and linear functions), and thus the solutions of the power allocation problems $\mathbf{P} \mathbf{A}_{F D}$ and $\mathbf{P A} H D$ can be easily found by using any convex optimization tools such as CVX [17]. After that we select the duplex mode with higher rate. Therefore, the solution of $\boldsymbol{P} 3$ can be obtained by applying the previous procedure on each pair $\left(D 2 D_{j}, C U_{j}\right)$. Since SCO is an iterative based solution and it require solving an approximated convex problem at each iteration, in the next section we look to provide a closed-form suboptimal solution and also find the upper bound condition for using FD mode.

\section{THE PROPOSED POWER ALLOCATION SCHEME}

Despite the interest of the SCO solution, the latter does not provide a closed-form analytical expression for the optimal power allocation and also may require high iteration numbers in some situation. Thus we use the SCO for benchmark purpose, and we present in this section a suitable analytical solution for $\boldsymbol{P 3}$. The proposed solution finds the optimal power ratio between the D2D users in the SIR domain and then calculates the corresponding power for each user in the SINR domain. The simulation results in section IV shows that the performance of the proposed technique is within the $95 \%$ of the benchmark results achieved by the SCO method but with much lower complexity. Indeed, the proposed solution finds a solution for the PA problem by only searching three points. In addition, the proposed solution provides valuable information about the necessary conditions to operate in FD mode.

\section{A. Optimal Power Ratio}

The aim of this subsection is to find the optimal power ratio between the D2D users. To that end, first we relaxed the power allocation problems $\mathbf{P A} F D$ and $\mathbf{P A} A_{H D}$ to $\mathbf{P A}{ }_{F D}$ and $\mathbf{P A}{ }_{H D}$ respectively by considering an interference limited system, i.e., the noise power is assumed to be negligible compared to the interference power.

$$
\begin{aligned}
& \mathbf{P A}_{F D}: \max _{\mathbf{p}_{j}} \log _{2}\left(Q_{f d}(x, y)\right) \text { s.t. } \mathbf{p}_{j} \in \Omega \\
& \mathbf{P A}_{H D}: \max _{\mathbf{p}_{j}} \log _{2}\left(Q_{h d}(x, y)\right) \text { s.t. } \mathbf{p}_{j} \in \Omega
\end{aligned}
$$

$$
\begin{aligned}
& Q_{f d}(x, y)=\left(1+\frac{g_{d} y}{g_{c, d 1}+\eta x}\right)\left(1+\frac{g_{d} x}{g_{c, d 2}+\eta y}\right) \\
& Q_{h d}(x, y)=\left(1+\frac{g_{d} y}{g_{c, d 1}}\right)\left(1+\frac{g_{d} x}{g_{c, d 2}}\right) \\
& \Omega=\left\{(x, y): g_{c, B S} \geq \gamma_{\min }^{c}\left(x g_{d 1, b s}+y g_{d 2, b s}\right)\right. \\
& \left.0 \leq x \leq M_{x}=\frac{g_{c, B S}}{\gamma_{\min }^{c} g_{d 1, B S}}, 0 \leq y \leq M_{y}=\frac{g_{c, B S}}{\gamma_{\min }^{c} g_{d 2, B S}}\right\}
\end{aligned}
$$

where $x=P_{d 1} / P_{c}$ and $y=P_{d 2} / P_{c}$ are the power ratio between the D2D users and the cellular user. $M_{x}$ and $M_{y}$ denote the maximum allowed power ratio which guarantees the CUE's QoS, i.e., $\gamma_{\text {min }}^{c} . \Omega$ stands for the feasible set of $\mathbf{P A}_{F D}$ and $\mathbf{P A}_{H D}$ and it can be illustrated as in Fig 2. The vertices $o, v_{1}$ and $v_{2}$ respectively represents the power ratio pairs $(0,0),\left(M_{x}, 0\right)$, and $\left(0, M_{y}\right)$. The lines $\overline{o v_{1}}, \overline{o v_{2}}$, and $\overline{v_{1} v_{2}}$ in Fig. 2 are the border lines of $\Omega$.

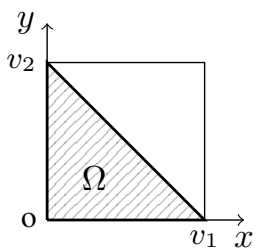

Fig. 2. Graphically illustration of the feasible set of $\boldsymbol{P 2}$.

In order to find the optimal power ratios of (18) and (19) we first provide Lemma 1 , and then we introduce Theorem 1.

Lemma 1. The optimal power ratio of $\boldsymbol{P A}^{\boldsymbol{A}_{F D}}$ or $\boldsymbol{P \boldsymbol { A } ^ { \prime }}{ }_{H D}$ must reside on the border line $\overline{v_{1} v_{2}}$.

Proof: First we provide the proof for the FD mode. For any scaling parameter $\lambda>1$ and a power ratio pair $(x, y) \in \Omega$ we have the following,

$$
Q_{f d}(\lambda x, \lambda y)=\log _{2}\left[\left(1+\frac{g_{d} y}{\frac{g_{c, d 1}}{\lambda}+\eta x}\right)\left(1+\frac{g_{d} x}{\frac{g_{c, d 2}}{\lambda}+\eta y}\right)\right] \geq Q_{f d}
$$

Equation (24) states that given any $(x, y)$ in the interior of the feasible region $\Omega$, one can always find another power ratio, $(\lambda x, \lambda y)$, that yields to a larger rate. Accordingly, the optimal point must lie on the border lines of $\Omega$. It is easily verified that the maximum of (18) on the lines $\overline{O v_{1}}$ and $\overline{o v_{2}}$ are respectively the vertices $v_{1}$ and $v_{2}$. Thus, the optimal point of (18) must reside on the line $\overline{v_{1} v_{2}}$. A similar proof can be derive for the HD mode.

Denote by $v_{f d}^{*}$ and $v_{h d}^{*}$ the optimal power ratio of (18) and (19) respectively. In the following theorem we show that both $v_{f d}^{*}$ and $v_{h d}^{*}$ can be found by only searching three points on the line $\overline{v_{1} v_{2}}$

Theorem 1. The optimal power ratios $v_{f d}^{*}$ and $v_{h d}^{*}$ belongs to the sets $\left\{v_{1} ; v_{2} ; v_{3}^{f d}\right\}$ and $\left\{v_{1} ; v_{2} ; v_{3}^{h d}\right\}$ respectively, where the points $v_{3}^{f d}$ and $v_{3}^{h d}$ lie on the line $\overline{v_{1} v_{2}}$.

Proof: Due to the monotonicity property of the logarithm, maximizing (18) or (19) is equivalent to maximizing $Q_{f d}(x, y)$ or $Q_{h d}(x, y)$ respectively. From Lemma 1 we know that the optimal point must resides on the line $\overline{v_{1} v_{2}}$. Now, applying the equality case of (22), i.e., the line $v_{1} v_{2}$, in $Q_{f d}(x, y)$ 
and $Q_{h d}(x, y)$ and differentiating the result w.r.t $x$ yields the following

$$
\begin{gathered}
\frac{\partial Q_{f d}(x)}{\partial x}=\frac{A x^{2}+2 B x+C}{F}, \\
\frac{\partial Q_{h d}(x)}{\partial x}=\left.\frac{\partial Q_{f d}(x)}{\partial x}\right|_{\eta=0},
\end{gathered}
$$

where

$$
\begin{aligned}
& A=g_{d} \eta\left(g_{c, d 2}+\eta M_{y}\right)\left(\eta-\beta g_{d}\right) \text {, } \\
& B=\left(g_{c, d 2}+\eta M_{y}\right)\left[M_{y} g_{d} \beta \eta^{2}+\eta g_{c, d 1} g_{d}+g_{c, d 1} \beta g_{d}\left(\eta \beta-g_{d}\right)\right] \text {, } \\
& C=\left(g_{c, d 2}+\eta M_{y}\right)\left[g_{c, d 1}{ }^{2} g_{d}+M_{y} g_{c, d 1} g_{d}^{2}\right. \\
& \left.-\left(g_{c, d 2}+\eta M_{y}\right)\left(\eta M_{y} g_{d}+g_{c, d 1} \beta g_{d}\right)\right], \\
& F=\left(\eta x+g_{c, d 1}\right)^{2}\left(\eta \beta x-g_{c, d 2}-\eta M_{y}\right)^{2} \text {, } \\
& \beta=g_{d 1, b s} / g_{d 2, b s}
\end{aligned}
$$

Since $F$ is seen to be always positive, the possible optima of $Q_{f d}$ can be found as the solution to $A x^{2}+2 B x+C$, which is given by,

$$
v_{3}^{f d}=\left(\frac{1}{A}\left(-B \pm \sqrt{B^{2}-A C}\right), M_{y}-\beta x\right)
$$

The obtained point, $v_{3}^{f d}$, does not always guarantee a feasible solution (since the solution of (27) may be an imaginary point or it may lie outside $\overline{v_{1} v_{2}}$ ) and does not always present a global maxima on the line $\overline{v_{1} v_{2}}$ (since there is no guarantee that the second derivative is always negative). Thus, using Lemma 1, the global maximum of (18) is either one of the corner points, i.e., $v_{1}$ or $v_{2}$, or $v_{3}^{f d}$ (if $v_{3}^{f d}$ is feasible).

For the HD mode, it is easily to see that the second derivative of $Q_{h d}$ is always negative. Thus, the optimal solution of $Q_{h d}$ is the solution of (26) which is equal to $v_{3}^{h d}=\left.\frac{-C}{2 B}\right|_{\eta=0}$. However, $v_{3}^{h d}$ is not always a feasible solution because it may reside outside $\overline{v_{1} v_{2}}$ and thus the solution of $Q_{h d}$ is also either $v_{1}$ or $v_{2}$ or $v_{3}^{h d}$ (if $v_{3}^{h d}$ is feasible).

This theorem shows that the optimal power ratios in the SIR domain $v_{f d}^{*}$ and $v_{h d}^{*}$ are reduced to a set of three points. The equivalent power of each user in the SINR domain is derived in the next subsection.

\section{B. Optimal transmission power and duplex mode}

Here, we are interested in finding a suitable sub-optimal solution of $\mathbf{P} \mathbf{A}_{F D}$ and $\mathbf{P A} \mathbf{A}_{H D}$ by using the optimal power ratio derived in the previous subsection. To that end, first we change the form of the the QoS constraint defined in (10) as follows:

$$
P_{c} \geq \frac{\gamma_{\min }}{g_{c, b s}}\left(g_{d 1, b s} P_{d 1}+g_{d 2, b s} P_{d 2}+N_{0}\right)
$$

By inspecting the objective function of $\mathbf{P A} F D$ and $\mathbf{P} \mathbf{A}_{H D}$, it is clear that the maximum rate occurs only if $P_{c}$ is at its minimum level. In other words, to obtain the maximum D2D rate the equality in (28) has to be achieved. Now by using this information, the corresponding transmission powers in the three dimensional space $\left(P_{d 1}, P_{d 2}, P_{c}\right)$, of $v_{1}, v_{2}, v_{3}^{f d}$, and $v_{3}^{h d}$ denoted respectively by $V_{1}, V_{2}, V_{3}^{f d}$, and $V_{3}^{h d}$ can be calculated as follows:

$$
\begin{gathered}
\mathbf{V}_{1}=\left(\min \left\{P_{\max }^{d 1}, M_{x}\left(P_{\max }^{c}-\mu N_{0}\right)\right\} ; 0 ; \mu\left(g_{d 1, b s} P_{d 1}^{\mathbf{V}_{1}}+N_{0}\right)\right) \\
\mathbf{V}_{2}=\left(0 ; \min \left\{P_{\max }^{d 2}, M_{y}\left(P_{\max }^{c}-\mu N_{0}\right)\right\} ; \mu\left(g_{d 2, b s} P_{d 2}^{\mathbf{V}_{2}}+N_{0}\right)\right)(30) \\
\mathbf{V}_{3}^{i}=\left(\operatorname { m i n } \left\{P_{\max }^{d 1}, \frac{\theta_{i}}{\mu\left(g_{d 2, b s}+\theta_{i} g_{d 1, b s}\right)}\left(P_{\max }^{c}-\mu N_{0}\right) ;\right.\right. \\
\min \left\{P_{\max }^{d 2}, \frac{1}{\mu\left(g_{d 2, b s}+\theta_{i} g_{d 1, b s}\right)}\left(P_{\max }^{c}-\mu N_{0}\right) ;\right. \\
\left.\mu\left(g_{d 1, b s} P_{d 1}^{\mathbf{v}_{3}^{i}}+g_{d 2, b s} P_{d 2}^{\mathbf{V}_{3}^{i}}+N_{0}\right)\right), i \in\{f d, h d\}
\end{gathered}
$$

where $\theta_{f d}=\frac{x^{v_{3}^{f d}}}{y^{v_{3}^{f d}}}, \theta_{h d}=\frac{x^{v_{3}^{h d}}}{y^{v_{3}^{h} d}}$ and $\mu=\frac{\gamma_{\min }^{c}}{g_{c, \mathrm{BS}}}$. Note that, $x^{t}$ and $y^{t}$ denotes the $\mathrm{x}$ and $\mathrm{y}$ abscissa of the power ratio point $t$. Similarly, $P_{k}^{t}, k \in\{d 1, d 2, c\}$, denotes the $P_{k}$ abscissa of the power point $t$.

Let $\mathbf{p}_{f d}$ and $\mathbf{p}_{h d}$ be the sub-optimal solution of (18) and (19) respectively. Then, similar to theorem $1, \mathbf{p}_{f d} \in$ $\left\{\mathbf{V}_{1}, \mathbf{V}_{2}, \mathbf{V}_{3}^{f d}\right\}$ and $\mathbf{p}_{h d} \in\left\{\mathbf{V}_{1}, \mathbf{V}_{2}, \mathbf{V}_{3}^{h d}\right\}$. Now, denote by $\underline{\mathbf{p}}^{*}=\max \left(\left.R_{f d}\right|_{\mathbf{p}=\mathbf{p}_{f d}},\left.R_{h d}\right|_{\mathbf{p}=\mathbf{p}_{h d}}\right)$ the sub-optimal solution of $\overline{\boldsymbol{P}}$ 3, the following theorem summarizes all the results.

Theorem 2. $\boldsymbol{p}^{*}$ can be found by only searching a set of four points as follows:

$$
\underline{\boldsymbol{p}}^{*}=\max \left(\left.R_{f d}\right|_{\boldsymbol{p}=\boldsymbol{V}_{1}},\left.R_{f d}\right|_{\boldsymbol{p}=\boldsymbol{V}_{2}},\left.R_{f d}\right|_{\boldsymbol{p}=\boldsymbol{V}_{3}^{f d}},\left.R_{h d}\right|_{\boldsymbol{p}=\boldsymbol{V}_{3}^{h d}}\right)
$$

Proof: It is easily verified that $R_{f d}$ is always greater to $R_{h d}$ when $\mathbf{p}$ is equal to $\mathbf{V}_{1}$ or $\mathbf{V}_{2}$, and thus only four points has to be searched instead of six. This is because the points $\mathbf{V}_{1}$ and $\mathbf{V}_{2}$ represent the case where only one of the D2D users is transmitting. Hence, in such case, the total bandwidth $B$ must be used by the transmitter instead of using half of the bandwidth.

As a result, a suitable solution of $\boldsymbol{P 3}$ can be found by applying (32) on each couple $\left(D 2 D_{j}, C U_{j}\right)$. Here we should strengthen that the solution derived in this section is highly tractable as it has much lower complexity than the SCO solution.

\section{Upper bound condition for using FD mode}

First, we observe that the D2D pair operates in FD mode only when $\mathbf{p}=\mathbf{V}_{3}^{f d}$ or equivalently when the power ratio is equal to $v_{3}^{f d}$. Thus, analyzing the performance of the $\mathrm{D} 2 \mathrm{D}$ rate at this point may lead to an important characteristics of FD. Here we are interesting in finding the upper bound condition of using FD mode, and thus we study the case of perfect SI cancellation $(\eta=0)$ in the SIR domain. In such case, $v_{3}^{f d}$ is reduced to the follows:

$$
v_{3}^{f d}=\left(\frac{M_{y} g_{d}+g_{c, d 1}-\beta g_{c, d 2}}{2 \beta g_{d}}, \frac{M_{y} g_{d}+\beta g_{c, d 2}-g_{c, d 1}}{2 g_{d}}\right)
$$

Moreover, when no RSI exist the second derivative of $Q_{f d}(x, y)$ can be written as

$$
\frac{\partial \partial Q_{f d}}{\partial \partial x}=\frac{2 B}{F}=\frac{-2 g_{c, d 1} g_{c, d 2} g_{d}^{2}}{F}<0 .
$$


From (34), it is clear that in the case of perfect SI cancellation (18) is always concave. Thus, the FD mode will be the optimal transmission mode only if $v_{3}^{f d}$ given in (33) is feasible and lies on $\overline{v_{1} v_{2}}$. Hence, by comparing (33) with $v_{1}$ and $v_{2}$ the optimal condition of FD mode can be written as

$$
\left|\frac{\gamma_{\min }^{c}\left(g_{d 1, \mathrm{BS}} g_{c, d 2}-g_{d 2, \mathrm{BS}} g_{c, d 1}\right)}{g_{c, \mathrm{BS}} g_{d}}\right|<1
$$

Accordingly, the D2D pair can operate in FD only when (35) holds true.

\section{NUMERICAL RESULTS}

We consider a single cell network with radius $R$ and $M$ CUEs and D2D pairs. The CUEs are uniformly located in the cell while each D2D pairs is uniformly located within a randomly located cluster with radius $r$. The different simulation parameters are summarized in Table I.

TABLE I

SIMULATION PARAMETERS

\begin{tabular}{|l|l|}
\hline Cell radius $(\mathrm{R})$ & $280 \mathrm{~m}$ \\
\hline Cluster radius $(\mathrm{r})$ & $10 \mathrm{~m}, 20 \mathrm{~m}, 40 \mathrm{~m}$ \\
\hline Noise power $\left(N_{0}\right)$ & $-114 \mathrm{dBm}$ \\
\hline Path-loss exponent $(\alpha)$ & 3.5 \\
\hline Maximum power of CUE and D2D users & $24 \mathrm{dBm}$ \\
\hline CUE SINR $\left(\gamma_{\min }^{c}\right)$ & $\begin{array}{l}\text { uniform distributed in } \\
{[0,25] \mathrm{dB}}\end{array}$ \\
\hline Bandwidth of $C U_{j}(B)$ & $180 \mathrm{KHz}$ \\
\hline Multiple-path fading & $\begin{array}{l}\text { Exponential distribu- } \\
\text { tion with mean } l_{i j}^{-\alpha}\end{array}$ \\
\hline
\end{tabular}

Fig. 3 compares the proposed power allocation scheme with the SCO method for different cluster radius and different SI cancellation factors. In this figure we generate $M=10$ CUEs and D2D pairs 200 times. Each iteration we averaged the results of all the couples $\left(D 2 D_{j}, C U_{j}\right)$ and finally we averaged the results of all the iterations. As it can be seen, the curves are very close for almost all the cases. This is because our approach finds the global optima in the SIR domain, so at high SINR regime the solutions will be almost the same. At low SINR regime the D2D rate will be very low and thus the difference between the two solution will not be that important. The main difference between the two solutions are at the medium SINR region. Moreover, Fig. 3 clearly shows that as the cluster radius $r$ increases the average rate decreases. In addition, we can see that the average rate increases as the SI factor decreases. For a SI coefficient $\eta<-70 d B$, i.e. for a good SI cancellation technique, the average rate is saturated. This is because at low SI, the interference terms of other users will play the major role in setting the FD rates as stated in (35).

In Table II we report the average number of iteration required for convergence for the SCO method. As it can be seen, on average SCO requires three to five iterations to achieve the solution. In addition, at each iteration $\mathrm{SCO}$ requires using a convex optimization solver to solve the approximated convex problem. On contrary, the proposed method can find a

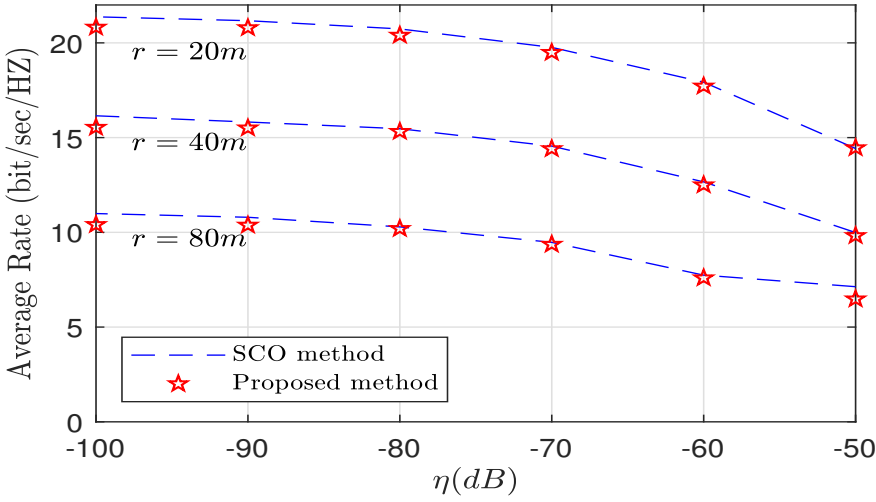

Fig. 3. Comparison of FD-D2D rate obtained from the exhaustive search and from the proposed power allocation scheme.

good solution with only one iteration and without using any optimization solver.

TABLE II

AVERAGE NUMBER OF ITERATION FOR THE SCO METHOD

\begin{tabular}{ccc}
\hline$\eta(\mathrm{dB})$ & \multicolumn{2}{c}{ Number of Iteration } \\
\cline { 2 - 3 } & $r=20 m$ & $r=40 m$ \\
\hline-50 & 2.41 & 2.28 \\
-60 & 2.45 & 2.35 \\
-70 & 2.59 & 2.41 \\
-80 & 3.34 & 2.88 \\
-90 & 4.98 & 3.79 \\
-100 & 6.15 & 5.42 \\
\hline
\end{tabular}

To show the effect of the CUE location on the optimal transmission powers we first set $D 1$ and $D 2$ at a distance equal to $100 \mathrm{~m}$ and $140 \mathrm{~m}$ from the BS. Then we moved the CUE toward the D2D pair and at each step we computed the optimal transmission powers as shown in Fig. 4. From Fig. 4 we can see that when the CUE is near the BS and far from the D2D pair $\left(d_{c, B S}=20 m\right)$ both $D 1$ and $D 2$ can transmit with their maximum powers. However, when the CUE becomes close to $D 1$ the transmission power of $D 2$ decreases and at the point $d_{c, B S}=70 \mathrm{~m}$ only $\mathrm{D} 1$ must transmit to achieve the optima. This is because starting from this point $D 1$ will face high interference from the $C U E$ and thus it is better to send messages rather than receiving messages. The same analogy can be applied to the case where the CUE is closer to D2. On the other hand, $P_{c}$ increases with the increasing of $d_{c, B S}$. This is because the CUE has QoS constraint. As a result, the CUE location highly affects the optimal mode selection.

To highlight the effect of the CUE location on the optimal D2D transmission mode, we reset the D2D users at $100 \mathrm{~m}$ and $140 \mathrm{~m}$ from the BS. Then, we moved the CUE on a random trajectory which covers almost all the cell. At each position we computed the optimal D2D transmission mode and we colored the CUE's location accordingly. As expected, when the CUE is far from the D2D pair, the yellow area in Fig. 5, the optima is achieved by using the FD mode. However, when the $C U E$ is close to the D2D pair, the blue sector in Fig 5, the optima can be achieved by turning off one of the D2D users. 


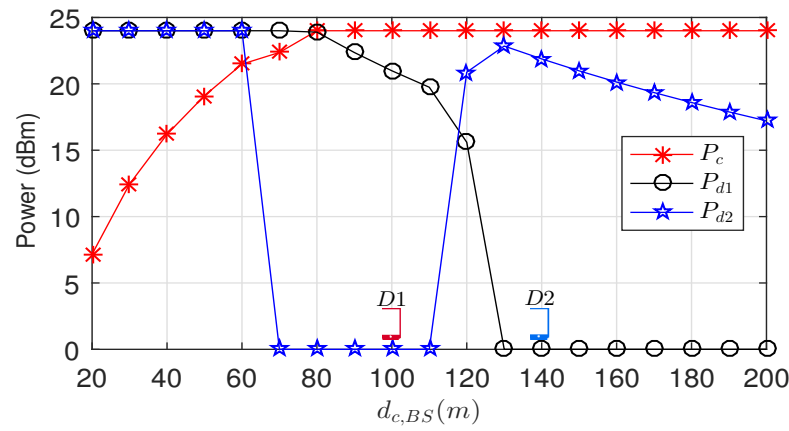

Fig. 4. The effect of the $C U E$ location on the optimal power $(\eta=-70 d B)$.

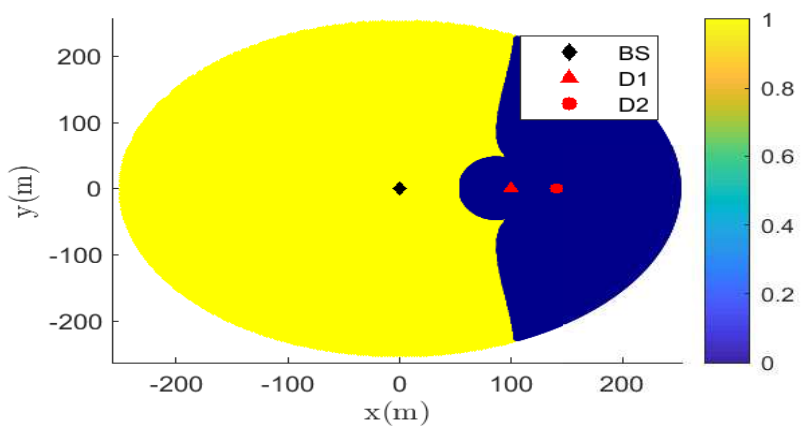

Fig. 5. The effect of the $C U E$ location on the optimal D2D transmission mode $(\eta=-70 d B)$.

\section{CONCLUSION}

In this paper we propose two frameworks to solve the joint mode selection and power allocation problem of a FD-D2D network. The first framework yields to a first-order optimal solution by using the SCO theory. The second framework gives a sub-optimal solution by finding the optimal power ratio between the users in the SIR domain and then computing the corresponding power in the SINR domain. The derived approach has been validated in simulation results. Among the different results, it is important to highlight the importance of the CUE location on the D2D transmission mode (HD or FD) as well the capabilities of the D2D devices to reduce the self-interference in the FD mode. As a future work, the maximization problem of the total rate, i.e., the rate of both cellular users and D2D users, will be considered. In addition, it might be interested to study the channel assignment problem of a FD-D2D network.

\section{ACKNOWLEDGEMENT}

This work was partially funded through French National Research Agency (ANR) project WONG5 with grant agreement code: ANR-15-CE25-0005603. A part of this work was also carried out thanks to the STSM grant of COST Action CA15104 (IRACON) and CNRS (GdR-ISIS).

\section{REFERENCES}

[1] V. Cisco Mobile, "Cisco visual networking index: Global mobile data traffic forecast update, 2016-2021 white paper," 2017.
[2] K. Doppler, M. Rinne, C. Wijting, C. B. Ribeiro, and K. Hugl, "Deviceto-device communication as an underlay to lte-advanced networks," IEEE Communications Magazine, vol. 47, no. 12, 2009.

[3] A. Sabharwal, P. Schniter, D. Guo, D. W. Bliss, S. Rangarajan, and R. Wichman, "In-band full-duplex wireless: Challenges and opportunities," IEEE Journal on Selected Areas in Communications, vol. 32, no. 9, pp. 1637-1652, 2014

[4] V. Tapio, "System scenarios and technical requirements for full-duplex concept," DUPLO, Project Deliverable, 2013.

[5] W. Cheng, X. Zhang, and H. Zhang, "Optimal power allocation for fullduplex d2d communications over wireless cellular networks," in 2014 IEEE Global Communications Conference, Dec 2014, pp. 4764-4769.

[6] B. Zuo, L. Jiang, C. He, and Z. Lian, "Power allocation optimization for full-duplex $\mathrm{d} 2 \mathrm{~d}$ communications underlaying cellular networks," in 2016 International Conference on Networking and Network Applications (NaNA). IEEE, 2016, pp. 103-108.

[7] K. T. Hemachandra, N. Rajatheva, and M. Latva-aho, "Sum-rate analysis for full-duplex underlay device-to-device networks," in 2014 IEEE Wireless Communications and Networking Conference (WCNC), April 2014, pp. 514-519.

[8] C. Liu, T. Lv, W. Tang, H. Gao, and Y. Lu, "On the performance of mode selection for hybrid-duplex d2d communications," in Proc. MILCOM 2017 - 2017 IEEE Military Communications Conf. (MILCOM), Oct. 2017, pp. 852-857.

[9] A. N. Kadhim, F. Hajiaghajani, and M. Rasti, "On selecting duplex-mode and resource allocation strategy in full duplex d2d communication," in Proc. Iranian Conf. Electrical Engineering (ICEE), May 2017, pp. 1640-1645.

[10] L. Han, W. Zou, and G. Zhao, "Duplex mode selection for device-todevice communications underlaying the cellular uplink," in Proc. IEEE Conf. Computer Communications Workshops (INFOCOM WKSHPS), May 2017, pp. 157-162.

[11] L. Venturino, A. Zappone, C. Risi, and S. Buzzi, "Energy-efficient scheduling and power allocation in downlink OFDMA networks with base station coordination," IEEE Transactions on Wireless Communications, vol. 14, no. 1, pp. 1-14, Jan. 2015.

[12] M. Chiang, C. W. Tan, D. P. Palomar, D. O'neill, and D. Julian, "Power control by geometric programming," IEEE Transactions on Wireless Communications, vol. 6, no. 7, pp. 2640-2651, Jul. 2007.

[13] L. Venturino, N. Prasad, and X. Wang, "Coordinated scheduling and power allocation in downlink multicell OFDMA networks," IEEE Transactions on Vehicular Technology, vol. 58, no. 6, pp. 2835-2848, Jul. 2009.

[14] T. Riihonen, S. Werner, and R. Wichman, "Mitigation of loopback selfinterference in full-duplex mimo relays," IEEE Transactions on Signal Processing, vol. 59, no. 12, pp. 5983-5993, 2011.

[15] A. Zappone, E. Björnson, L. Sanguinetti, and E. Jorswieck, "Globally optimal energy-efficient power control and receiver design in wireless networks," IEEE Transactions on Signal Processing, vol. 65, no. 11, pp. 2844-2859, 2017.

[16] B. Matthiesen and E. A. Jorswieck, "Global sum rate optimal resource allocation for non-regenerative 3-way relay channels," in Ubiquitous Wireless Broadband (ICUWB), 2017 IEEE 17th International Conference on. IEEE, 2017, pp. 1-5.

[17] M. Grant, S. Boyd, and Y. Ye, "Cvx: Matlab software for disciplined convex programming," 2008. 